\title{
Mecanismo de Acesso Inicial baseado em Geolocalização para Redes de Ondas Milimétricas
}

\author{
Alex Lambiase Schramm ${ }^{1}$, Marcel William Rocha da Silva ${ }^{2}$, \\ José Ferreira de Rezende ${ }^{1}$ \\ ${ }^{1}$ COPPE - Universidade Federal do Rio de Janeiro (UFRJ) \\ ${ }^{2}$ DCC - IM - Universidade Federal Rural do Rio de Janeiro (UFRRJ) \\ alexschrammecos.ufrj.br, marcelsilva@ufrrj.br, rezende@land.ufrj.br
}

\begin{abstract}
The use of directional antennas in frequency bands above $10 \mathrm{GHz}$ becomes mandatory due to the high attenuation and other propagation phenomena that occur in these bands. Therefore, user's initial access in cellular networks of this type becomes slower because it requires that the base station and the user do beamforming before communication. The use of user's positioning information is a possible way to speed up this procedure, but so far no work has considered the impact of geolocation errors in this process. In this work, we propose two new initial access mechanisms aware of the geolocation errors, which explore a greater angular space to find combinations of beam with improved performance.

Resumo. O uso de antenas diretivas em faixas de frequência acima de $10 \mathrm{GHz}$ torna-se obrigatório devido à alta atenuação e outros fenômenos de propagação que ocorrem nessas bandas. Por conseguinte, o acesso inicial de usuários a redes celulares desse tipo se torna lento pois exige-se que a estação base e o usuário façam beamforming antes que possam se comunicar. Uma alternativa para tornar mais rápido esse processo é pelo uso da informação de posicionamento do usuário, mas até o momento nenhum trabalho considerou o impacto de erros de geolocalização nesse processo. Neste trabalho, propõem-se dois novos mecanismos cientes dos erros de geolocalização que exploram um maior espaço angular para encontrar combinações de feixe com melhor desempenho.
\end{abstract}

\section{Introdução}

Nos últimos anos, vem crescendo a necessidade por novas tecnologias de comunicação sem fio que proporcionem um aumento da taxa de dados em redes celulares. No entanto, com a escassez de faixas de frequência abaixo de $10 \mathrm{GHz}$ [Rappaport et al. 2013], operar em ondas milimétricas $(\gg 10 \mathrm{GHz}$ ) passou a ser uma alternativa promissora, mesmo que existam grandes desafios em trabalhar com frequências tão elevadas [Rangan et al. 2014]. Com o comprimento de onda reduzido $(\ll 30 \mathrm{~mm})$, uma grande quantidade de materiais, tais como o corpo humano e até mesmo o oxigênio, causam uma alta atenuação do sinal, reduzindo fortemente o alcance de comunicação [Bai and Heath 2014].

Por outro lado, como o comprimento de onda é bastante reduzido, torna-se possível projetar antenas formadas por uma matriz com um grande número de elementos. Pelo uso dessas matrizes, pode-se criar vetores de apontamento orientáveis e estreitos, o que permite realizar transmissões altamente direcionais, amenizando os problemas de atenuação do sinal [Balanis 2016]. 
A matriz de elementos proporciona um grande número de possibilidades de escolha de feixe, tanto para a estação base milimétrica (EBmm) quanto para o usuário (USR), gerando uma grande quantidade de combinações possíveis de feixes entre ambos. O mecanismo que permite escolher a melhor combinação a cada instante é chamado de beamforming $(\mathrm{BF})$ e diversos trabalhos propõem diferentes estratégias para executar esse processo de escolha [Barati et al. 2015b, Giordani et al. 2016a, Giordani et al. 2016b].

Considerando-se que um USR em estado de repouso tem disponível um certo número de EBmm para se associar, denomina-se de acesso inicial (AI) o procedimento onde o USR descobre qual a melhor EBmm e estabelece a comunicação na camada de enlace [Barati et al. 2015a]. Esse procedimento nas redes de ondas milimétricas (ROmm) é bem mais custoso do que nos sistemas celulares atuais pois ele está diretamente relacionado ao mecanismo de BF. Além disso, devido à elevada intermitência do canal sem fio nessa banda, o USR deve realizar esse procedimento com uma certa frequência, tornando significativo o tempo gasto nesse processo, o que afeta diretamente a QoS do sistema.

Como os algoritmos de BF afetam diretamente a latência do acesso inicial [Barati et al. 2016], a investigação desses mecanismos é de grande importância. Um método de BF que serve de referência para outras propostas é o método exaustivo, onde o USR e a EBmm testam exaustivamente todas as combinações de feixes possíveis e escolhem aquela com maior relação sinal-ruído. A desvantagem desse método está relacionada ao tempo necessário para a realização desse processo que cresce com o aumento do número de feixes. Por outro lado, ele traz como principal vantagem a escolha da melhor combinação de feixes a cada instante. Ainda, a necessidade de se realizar periodicamente esse processo pode torná-lo inviável em algumas condições de canal. Para mitigar esse problema, uma outra classe de algoritmos leva em conta a informação de contexto [Giordani et al. 2016b], trocada por um canal de controle em micro-ondas. Nessa classe de mecanismos baseada em contexto, uma das formas mais estudadas é o uso do geoposicionamento [Giordani et al. 2016b, Capone et al. 2015, Peng et al. 2016].

Neste trabalho, analisamos o impacto do erro de geoposicionamento nos mecanismos de BF que se baseiam nessa informação para apressar o acesso inicial. Em seguida, propomos estratégias conscientes desses erros e as comparamos com mecanismos da literatura. Para realizar essa comparação, definimos uma metodologia, e uma métrica de desempenho associada, que permite capturar de forma justa o compromisso entre o tempo na escolha da combinação de feixes USR-EBmm e a relação sinal-ruído obtida.

O restante do artigo está organizado da seguinte forma. Na Seção 2, descrevemos os trabalhos relacionados ao problema de acesso inicial em redes de ondas milimétricas e as soluções por eles propostas. Na Seção 3, apresentamos nossas propostas de algoritmos baseados em contexto. Na Seção 4, detalhamos o modelo usado no simulador. Na Seção 5, analisamos nossos métodos em comparação aos outros, descrevendo a metodologia aplicada nas simulações e avaliando os resultados obtidos. Finalmente, na Seção 6, fazemos uma análise crítica dos esquemas propostos e apresentamos os trabalhos futuros.

\section{Trabalhos Relacionados}

Uma característica em comum a todos os trabalhos que estudam redes de ondas milimétricas é o fato de considerarem o uso da técnica de matrizes de antenas (antenna arrays) para o direcionamento e aumento do ganho do sinal transmitido. As matrizes de 


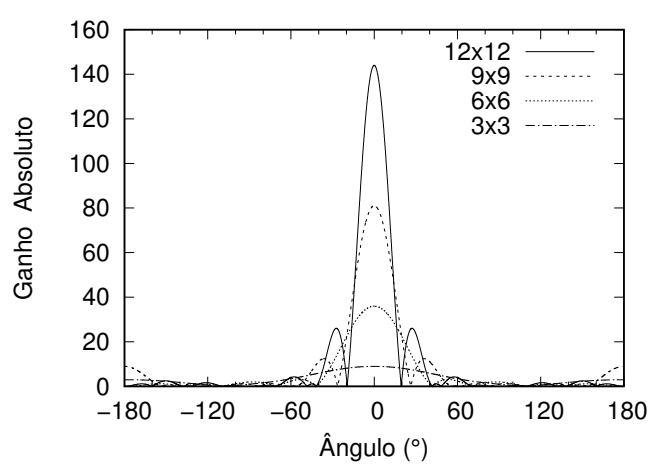

(a) Valores absolutos

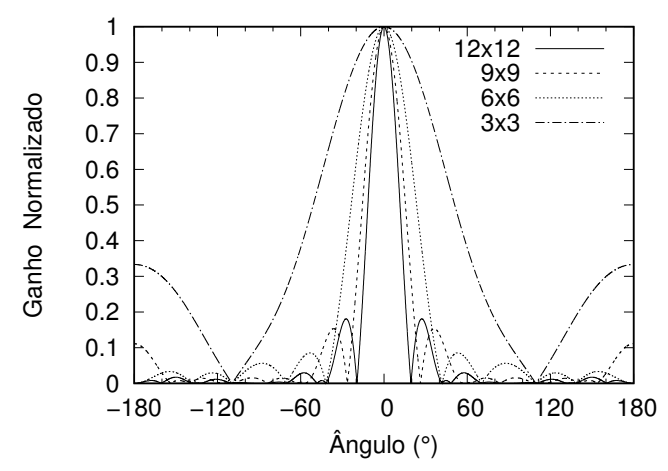

(b) Valores normalizados

Figura 1. Ganho de diferentes matrizes de antenas.

antenas são criadas através da junção de vários elementos irradiantes em uma disposição espacial no formato de matriz com uma distância uniforme entre os elementos. Estes elementos trabalham em conjunto como uma única antena que possui um padrão de irradiação próprio [Balanis 2016].

Para conseguir este efeito, cada elemento irradiante transmite o mesmo sinal com fases diferentes. Os efeitos construtivo e destrutivo desse conjunto de sinais irradiados pelos múltiplos elementos cria um padrão de irradiação diretivo e com alto ganho. Através do ajuste eletrônico da defasagem entre os sinais injetados em cada elemento irradiante, é possível modificar o padrão de irradiação do conjunto, fazendo com que seja possível modificar a direção do feixe de maior ganho (lóbulo principal). Neste contexto, considera-se a utilização de um livro de códigos (codebook) com conjuntos de defasagens pré-determinadas que geram feixes de irradiação em diferentes direções que cobrem todo o espaço angular do transmissor [Wei et al. 2017].

A quantidade de elementos irradiantes determina o ganho máximo que se obtém no lóbulo principal, o qual é dado pelo número de linhas multiplicado pelo número de colunas da matriz de antenas [Balanis 2016]. Os gráficos da Figura 1 apresentam o ganho de diferentes tamanhos de matrizes de antenas em função do ângulo de incidência para o caso em que o lóbulo principal da matriz está direcionado para o ângulo de zero grau. Pode-se perceber que a quantidade de elementos na matriz influencia tanto o ganho máximo quanto a largura do feixe. Assim, o emprego de matrizes de antenas permite modificar eletronicamente, sem elementos mecânicos [Balanis 2016], a direção do feixe de transmissão, viabilizando o uso de técnicas de BF.

Desta forma, diversos trabalhos propuseram algoritmos para a realização do BF considerando-se a utilização de uma matriz de antenas. Um algoritmo de referência é o da busca exaustiva [Wei et al. 2017] que consiste em uma varredura dos diferentes feixes presentes em seus codebooks para a transmissão de sondas de forma que todas as possíveis combinações de feixe da EBmm e do USR sejam testadas. A Figura 2 apresenta um exemplo de EBmm e USR, cada um com uma quantidade de feixes de transmissão próprios e uma matriz que contém todas as combinações de feixe a serem testadas. Apesar de ser a técnica que demanda mais tempo, a busca exaustiva tem a vantagem de ser a técnica capaz de encontrar a combinação de feixes da EBmm e USR que garante a maior relação sinal-ruído (SNR) nas comunicações posteriores. 


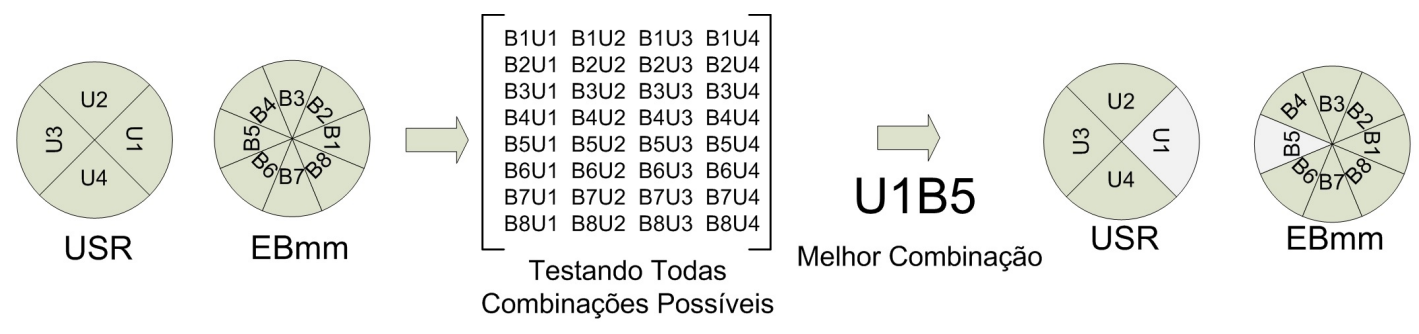

Figura 2. Exemplo de execução do algoritmo de busca exaustiva.

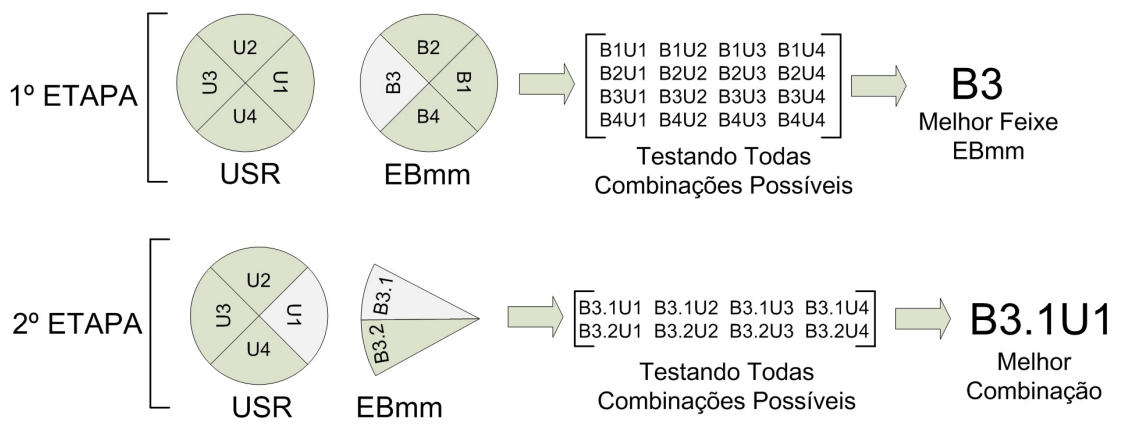

Figura 3. Exemplo de execução do algoritmo de busca iterativa.

Para reduzir o tempo despendido na busca, os autores em [Giordani et al. 2016a] propõem um algoritmo iterativo dividido em dois estágios. No primeiro, utiliza-se feixes de transmissão com maior abertura angular, que permitem fazer a varredura de todo o espaço angular com menos feixes. Numa segunda etapa, dentro do espaço angular em que se obteve maior SNR na primeira etapa, faz-se uma nova varredura utilizando os feixes de menor abertura. Os resultados mostram que o processo iterativo é mais rápido que o exaustivo. Apesar disso, deve-se ressaltar que o processo iterativo apresenta maior possibilidade de falha na detecção entre EBmm e USR, já que o feixe mais aberto utilizado na primeira etapa possui menor ganho em relação ao feixe mais estreito, o que pode tornar impossível a obtenção de uma SNR mínima para a detecção do sinal transmitido. A Figura 3 apresenta um exemplo de execução do algoritmo iterativo em cada etapa.

Outra classe de algoritmos é aquela que utiliza informações de contexto para auxiliar o processo de BF. A Figura 4 apresenta um cenário típico considerado pelos trabalhos que seguem esta linha. Neste cenário, considera-se que existe uma rede subjacente, que funciona como um canal de controle fora da banda, por onde EBmm e USR podem trocar informações e receber sinais de sincronização [Shokri-Ghadikolaei et al. 2015].

A informação de geolocalização, trocada pelo canal de controle, permite que o processo de BF seja realizado mais rapidamente. Em [Giordani et al. 2016b], os autores consideram que o USR envia sua geolocalização, obtida através de GPS, para a EBmm. Desta forma, o procedimento de BF para a EBmm se resume a calcular a direção em que o USR se encontra e escolher o feixe de transmissão naquela direção. Isso reduz enormemente o tempo necessário para o $\mathrm{BF}$ na $\mathrm{EBmm}$, a qual não necessita fazer varredura em diferentes combinações de feixe para a escolha da melhor combinação. Neste mesmo artigo, propõe-se uma variação desse método, ao qual demos o nome de GPS refinado, onde o EBmm faz uma varredura não somente no feixe de maior ganho, mas também nos seus dois feixes adjacentes. Um problema dos métodos propostos em [Giordani et al. 2016b] 


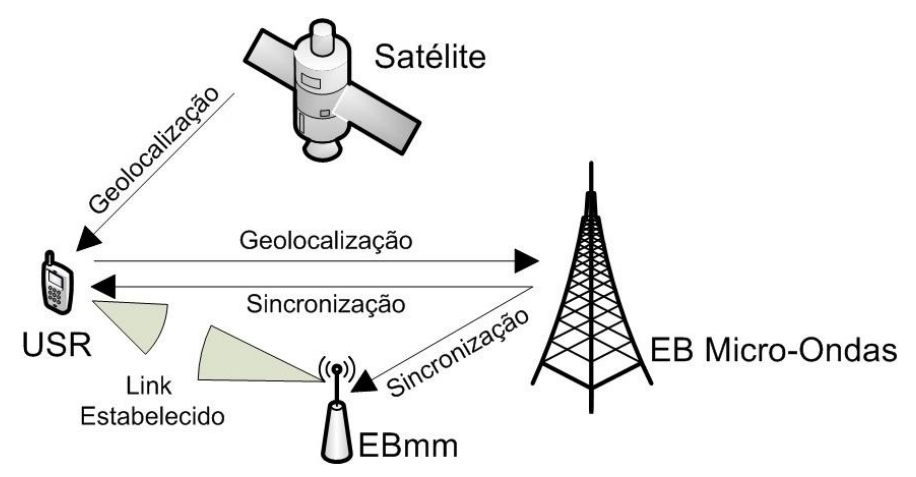

Figura 4. Funcionamento de um alg. de BF baseado em informações de contexto.

é que não se considera que a informação de geolocalização obtida através do GPS pode possuir um erro associado, o qual pode levar a conclusões erradas a respeito de qual é o melhor feixe a ser utilizado. Tais impactos serão melhor explicados na Seção 3.

Além do trabalho supracitado, outros usam informação de contexto em conjunto com técnicas de aprendizado de máquina, como em [Capone et al. 2015] e [Peng et al. 2016]. Estes trabalhos consideram que diversas informações de contexto a respeito dos USRs, como por exemplo, com qual EBmm o USR estabeleceu o enlace e quais foram os feixes utilizados, são armazenados em uma base de dados única. Em conexões futuras, essas informações são utilizadas pelo método de aprendizado para definir os melhores parâmetros a serem usados, buscando agilizar o acesso inicial com a maior SNR possível. Entretanto, se considerarmos efeitos como mobilidade dos USRs e dos obstáculos do cenário, tais informações tornam-se imprecisas rapidamente, causando a ineficácia das estratégias por aprendizado de máquina adotadas por esses trabalhos.

\section{Propostas}

Como descrito na seção anterior, as estratégias que utilizam a informação de geoposicionamento para apressar o acesso inicial não avaliaram o impacto da acurácia do sistema de geoposicionamento nas suas propostas. Nesta seção, utilizaremos uma abordagem geométrica para avaliar esse impacto em relação à disposição do receptor e do transmissor. A partir dessa avaliação, dois novos algoritmos conscientes desses erros foram propostos e serão descritos nesta seção.

Ambas as propostas usam a informação de posicionamento do usuário para a escolha do feixe principal e realizam uma varredura numa maior área angular para diminuir o impacto do erro nesse posicionamento, buscando o menor número de iterações possível para não onerar o tempo de busca. A primeira proposta reduz o tamanho da matriz de antenas e testa o feixe principal e seus adjacentes para ter uma maior cobertura com menos tempo de BF. Em seguida, refina o melhor feixe da primeira fase com feixes mais estreitos. A segunda proposta utiliza feixes estreitos de forma intercalada e seleciona os dois feixes com a melhor relação sinal-ruído para então testar seus feixes adjacentes.

Na próxima subseção, será feita uma avaliação do erro de geolocalização em função da distância entre USR e EBmm, para em seguida, apresentarem-se as duas propostas desse trabalho. 


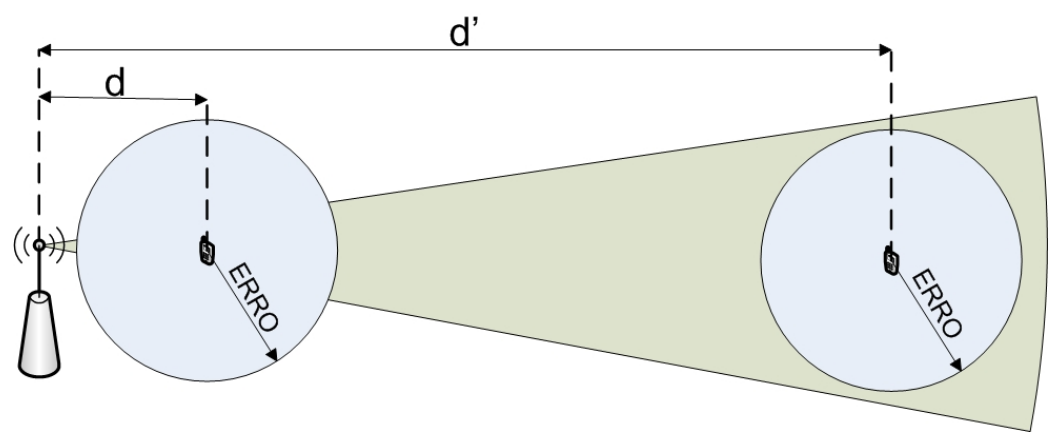

Figura 5. Conceito geométrico do erro de geoposicionamento.

\subsection{Impacto do Erro de Geoposicionamento no Acesso Inicial}

A informação correta do posicionamento do usuário permite à EBmm e ao USR realizar o BF de forma rápida e precisa [Giordani et al. 2016b]. No entanto, em áreas urbanas ou de florestas [Vaitl et al. 2010, Menard et al. 2011, Menard and Miller 2011], onde ocorre o bloqueio, reflexão ou difração do sinal [Renfro et al. 2018], o erro de geolocalização pode ser bastante elevado. Desta forma, o uso dessa informação pode determinar uma decisão não ótima, podendo levar a uma taxa de de transmissão reduzida ou até mesmo a falha na comunicação. Ao analisarmos geometricamente a distância entre EBmm e USR conjuntamente com o erro de geolocalização (Figura 5), podemos notar que quanto mais próximo está o USR da EBmm, maior é o impacto do erro. Quando o USR está a uma distância $d$ da EBmm, o raio do erro é muito mais significativo em relação à abertura do feixe, resultando em um erro angular máximo $\left(E A_{\max }\right)$, do que quando o USR está a uma distância $d^{\prime}$, onde o erro é menos relevante em proporção ao tamanho da abertura do feixe $\left(E A_{\max }^{\prime}\right)$. Ou seja, se $d<d^{\prime}$ então $E A_{\max }>E A_{\max }^{\prime}$.

Intuitivamente, isso significa que em distâncias menores, a varredura deve ser feita em um maior espaço angular do que em distâncias maiores. Por outro lado, maior espaço angular significa maior número de feixes e por conseguinte maior tempo de busca. Tais constatações nos levaram a desenhar as propostas descritas em seguida.

\subsection{GPS + Iterativo}

O primeiro algoritmo é dividido em duas etapas. Na fase inicial, a EBmm utiliza a posição do USR obtida do sistema de geoposicionamento para fazer a escolha do feixe principal e seus adjacentes, os quais serão usados durante o beamforming. Por outro lado, o usuário usa todos seus feixes, tentando obter a melhor SNR possível. Caso não consiga obter uma SNRmin, essa etapa deve ser refeita. Ao invés da EBmm usar uma matriz de antenas com todos os elementos para obter um maior ganho, ela utiliza uma matriz com um número menor de elementos, fazendo com que o feixe tenha uma maior abertura angular e assim realizar uma varredura mais ampla. Isso permite realizar um beamforming mais rápido, mesmo que isso cause uma redução significativa em seu ganho. Na fase final, o feixe da EBmm com o maior SNR da primeira etapa é dividido em sub-feixes de menor abertura e maior ganho, empregando-se toda a matriz de elementos. Então, realiza-se o beamforming entre os sub-feixes da EBmm e os feixes do USR, buscando a combinação com o maior SNR. A Figura 6 ilustra o funcionamento desse algoritmo. 


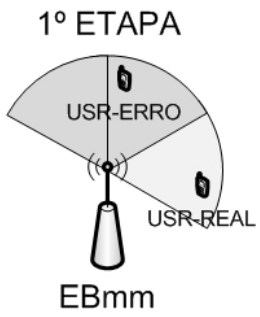

MATRIZ 3X3

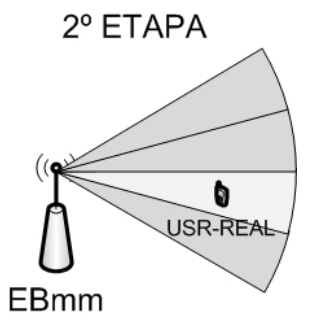

MATRIZ $12 X 12$

Figura 6. Representação do algoritmo GPS + Iterativo

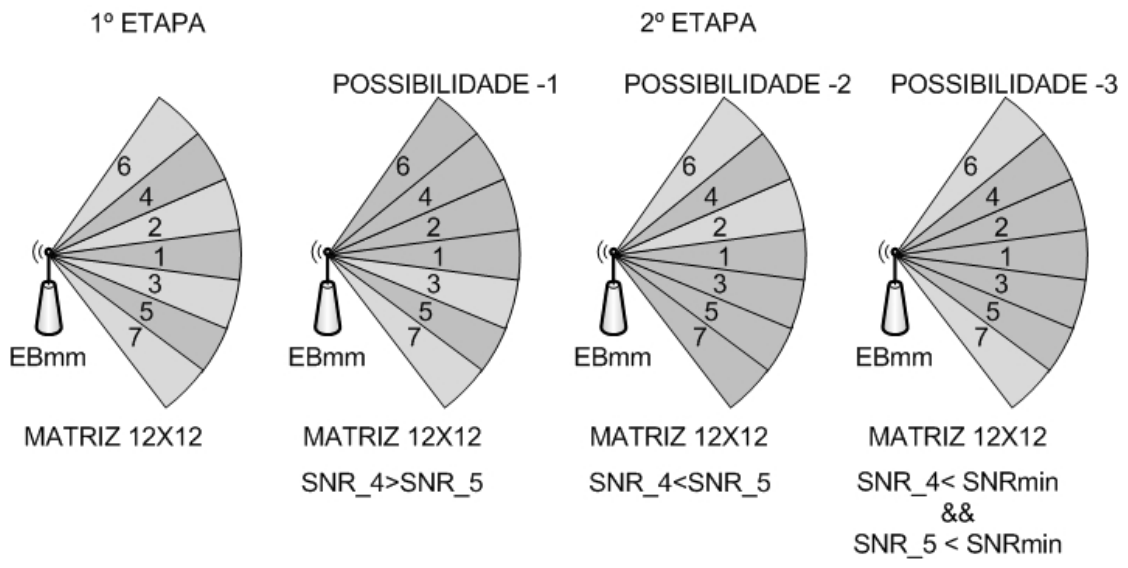

Figura 7. Representação do algoritmo Refinado + Busca

\subsection{Refinado + Busca}

O segundo algoritmo também possui duas fases. Inicialmente, a EBmm usa a posição do USR para realizar a escolha do feixe principal e de seus feixes adjacentes intercalados. Em seguida, é realizado o BF entre esses três feixes e os feixes do usuário, obtendo a melhor SNR para cada feixe da EBmm utilizado. Na fase seguinte, uma comparação das medidas de SNR obtidas nos feixes intercalados adjacentes é realizada. O feixe que obtiver a melhor SNR terá seus feixes adjacentes testados. No caso de não se obter nenhuma informação de SNR nesses feixes, pelo fato de não alcançarem um nível mínimo de sinal durante o BF, os dois feixes adjacentes do principal serão testados. Pela Figura 7, podemos entender melhor o comportamento da proposta. Nessa figura, o feixe principal é sempre numerado com $1, \operatorname{logo}$ os feixes 4 e 5 são os intercalados adjacentes. Na primeira possibilidade, o SNR do feixe 4 foi maior que o do 5, então os feixes 2 e 6 são testados. A segunda possibilidade é o inverso da primeira, então serão avaliados os feixes 3 e 7 . A última possibilidade corresponde ao caso de ambos os feixes 4 e 5 não terem obtido respostas de SNR, nesse caso os feixes 2 e 3 serão escolhidos para o BF. Ao final de todo o processo, a EBmm terá obtido informações de SNR de cinco feixes, assim como as combinações de feixes que as geraram. Para estabelecer a comunicação, será escolhida a combinação com maior SNR.

\section{Modelo}

Neste trabalho, desenvolvemos um novo modelo que fornece uma métrica única para avaliar o desempenho dos algoritmos de BF descritos nas seções anteriores. Esse modelo leva 


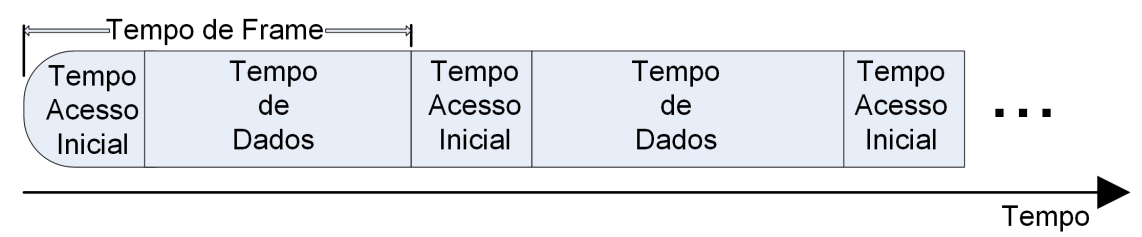

Figura 8. Modelo para a avaliação do acesso inicial em ROmm.

em consideração e integra duas métricas conflitantes: o tempo gasto no procedimento de acesso inicial $\left(t_{A I}\right)$ e a SNR do enlace estabelecido através desse procedimento. O tempo gasto no acesso inicial está diretamente relacionado ao tempo de execução do algoritmo de BF e representa uma sobrecarga (overhead) na comunicação entre EBmm e USR, enquanto a SNR obtida indica a eficiência do algoritmo de BF na busca por uma combinação de feixes EBmm-USR que forneça uma elevada qualidade de comunicação.

Usualmente, quanto maior o tempo gasto no procedimento de acesso inicial pelo algoritmo de BF, maior serão as chances do enlace formado possuir uma alta SNR, pois o algoritmo terá testado mais combinações de feixes entre EBmm e USR. Em contrapartida, algoritmos que demandam menos tempo no procedimento de acesso inicial têm a tendência a formar enlaces com menor SNR e consequentemente menor capacidade.

O modelo proposto assume que a execução do procedimento de acesso inicial é uma tarefa frequente e recorrente em uma ROmm. Tal característica pode ser causada na prática por diferentes fatores, dentre os quais se destaca a intermitência dos enlaces devido à grande variabilidade do canal em altas frequências [Barati et al. 2016]. Assim, o modelo considera que, do ponto de vista de um usuário, a comunicação com a EBmm ocorre em superquadros. como mostrado no diagrama da Figura 8 , os quais têm duração representada por um tempo de quadro $\left(t_{\text {Frame }}\right)$. Cada superquadro é dividido em duas fases. Na primeira, acontece a execução do procedimento de acesso inicial para o estabelecimento do enlace, com duração $t_{A I}$. Na segunda fase, ocorre a transmissão de dados, onde EBmm e USR poderão se comunicar pelo enlace estabelecido, com duração $t_{\text {Dados }}$.

A partir do modelo proposto pode-se derivar uma nova métrica de desempenho que é dada por uma combinação do tempo de acesso inicial e a SNR obtida na formação do enlace. Inicialmente, considera-se que o enlace recém formado no acesso inicial terá

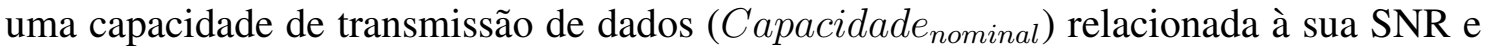
à sua largura de banda $B$ de acordo com a equação da capacidade do canal de Shannon (Equação 1) [Rappaport 1996].

$$
\text { Capacidade }_{\text {nominal }}=B \times \log _{2}(1+S N R)
$$

A partir da definição da Capacidade $_{\text {nominal }}$, e considerando o modelo proposto na Figura 8, pode-se definir a métrica de Capacidade $_{\text {fetiva }}$ conforme apresentado na Equação 2. Esta métrica de desempenho é dada pela Capacidade nominal $_{\text {ponderada pelo }}$ tempo disponível para a transmissão de dados no superquadro do modelo proposto. Assim, pode-se avaliar com uma única métrica de desempenho o impacto do tempo gasto com acesso inicial e a qualidade do enlace formado pelo método. 


$$
\text { Capacidade }_{\text {efetiva }}=\text { Capacidade }_{\text {nominal }} \times\left(\frac{t_{\text {Frame }}-t_{A I}}{t_{\text {Frame }}}\right)
$$

Esta modelagem e a métrica de desempenho proposta serão utilizadas para avaliar os algoritmos de acesso inicial baseados em informação de contexto propostos na Seção 3 em comparação com os algoritmos presentes na literatura.

\section{Avaliação de Desempenho}

Para avaliar o desempenho dos mecanismos de acesso inicial propostos, foi implementado um simulador próprio em linguagem $C$ que segue o modelo apresentado na Seção 4. O simulador emula um cenário em duas dimensões contendo uma EBmm e um USR em uma topologia estática, sem mobilidade dos equipamentos. Cada rodada de simulação representa um superquadro contendo as duas fases descritas no modelo: acesso inicial e transmissão de dados. A duração do superquadro $\left(t_{\text {Frame }}\right)$ é um dos parâmetros de entrada do simulador, o que permite avaliar qual o impacto da demanda por operações frequentes de acesso inicial para o reestabelecimento do enlace.

A implementação faz algumas considerações a respeito do funcionamento da ROmm semelhantes àquelas feitas em [Barati et al. 2016], o qual propõe um arcabouço de funcionamento na forma de uma extensão do utilizado em redes LTE. Desse trabalho, consideramos o fato de que o BF acontece através do envio de sondas (PSSs - Primary Synchronization Signals) por parte da EBmm, que são pacotes pequenos enviados em intervalos de tempo periódicos, $t_{p e r}$. A SNR dos pacotes recebidos é calculada pelo simulador, permitindo que os diferentes algoritmos possam comparar o desempenho das diversas combinações de feixes para a escolha daquele que fornece a melhor SNR.

Vale destacar que, para o completo funcionamento dos algoritmos de BF, devese considerar que existe algum tipo de resposta do USR para EBmm informando em qual combinação de feixe foi a escolhida durante o BF. Nesta implementação assumiu-se, conforme em [Shokri-Ghadikolaei et al. 2015], que existe uma macrocélula subjacente à ROmm que serve como canal de comunicação fora da banda por onde o USR notifica a EBmm da escolha realizada. Como escolha de implementação, assumiu-se que a quantidade de informação a ser enviada é pequena e o tempo de envio dessa resposta no canal fora da banda é desprezível.

Para representar a transmissão dos sinais com comprimento de ondas milimétricas, implementou-se no simulador um modelo de propagação que foi em parte baseado em [Akdeniz et al. 2014]. Nesse trabalho, os autores desenvolvem um modelo de propagação para ondas milimétricas baseado em experimentos reais de transmissão de sinais em altas frequências ( $28 \mathrm{GHz}$ e $73 \mathrm{GHz}$ ) na cidade de Nova Iorque nos EUA. Através da análise estatística dos resultados dos experimentos, o artigo propõe um modelo estatístico de perda de percurso em larga escala (path loss), o qual é capaz de determinar de acordo com a distância entre transmissor e receptor as probabilidades da comunicação acontecer em visada direta (LOS - Line of Sight), sem visada direta (NLOS - Non Line of Sight) ou da comunicação ser inviável (outage). Para cada tipo de comunicação, LOS ou NLOS, existe uma parametrização da expressão do cálculo da perda de percurso. Quando ocorre um outage, determinado pelo modelo, a perda no percurso é considerada infinita. 
Outro fenômeno importante para a propagação de sinais em ondas milimétricas é a variabilidade do canal determinada pelo desvanecimento em pequena escala ( $f a s t ~ f a-$ ding). Esse desvanecimento é causado não só pela mobilidade do receptor, mas também pela mobilidade do cenário e os efeitos da propagação por múltiplos percursos que varia em pequenos intervalos de tempo, resultando em interferências construtivas e destrutivas do sinal. Para representar essa variação ao longo do tempo de simulação no simulador implementou-se o modelo de Rayleigh, conforme descrito em [Rappaport 1996].

Além destas funcionalidades básicas para a avaliação de desempenho do processo de acesso inicial em ROmm, também foram implementados no simulador diferentes algoritmos de BF. Foi implementada a busca exaustiva (identificado como Exaustivo nos gráficos), conforme descrito na Seção 2. Implementou-se também o uso simples da informação da geolocalização (GPS) e a sua variante que verifica, além do feixe indicado pela geolocalização, os feixes adjacentes (GPS refinado). Em ambos os casos, conforme descrito em [Giordani et al. 2016b] e na Seção 2. E por fim, foram implementados também os dois novos algoritmos propostos na Seção 3, identificados como GPS+Iterativo e Refinado+Busca nos gráficos.

\subsection{Ambiente de simulação}

Para os resultados de simulação que serão apresentados a seguir foram realizadas simulações de 2 mil superquadros para cada distância entre EBmm e USR. Em cada cenário, o USR é posicionado em uma direção aleatória da EBmm, mas mantendo a distância desejada. As distâncias entre EBmm e USR foram variadas de 4 até 200 metros, em passos de incremento de 4 metros. Os resultados apresentados são as médias das métricas avaliadas com barras de erro correspondentes aos intervalos de confiança calculados com um nível de confiança de $95 \%$.

Um dos parâmetros variados nas simulações foi o tamanho do superquadro $\left(t_{\text {Frame }}\right)$. Conforme mencionado anteriormente, o tamanho do superquadro também pode ser interpretado como o intervalo entre operações de acesso inicial na ROmm. Cenários com $t_{\text {Frame }}$ pequenos visam representar cenários onde há maior intermitência do enlace causada pela alta variação do canal. Enquanto cenários com $t_{\text {Frame }}$ grandes visam representar cenários onde os enlaces estabelecidos pelo acesso inicial são mais estáveis. Serão apresentados resultados de simulação com valores de $t_{\text {Frame }}$ iguais à 200, 500 e 1500 ms.

Em todos os cenários de simulação a EBmm e o USR utilizam matrizes de antenas 12x12 e 4x4, respectivamente. Sendo que na fase inicial da proposta GP S+iterativo a EBmm utiliza um subconjunto $3 \times 3$ da matriz 12x12. Em todos os casos, a separação entre os elementos irradiantes é de $1 / 4$ do comprimento de onda $(\lambda / 4)$.

Para o cálculo da perda de percurso, utiliza-se os parâmetros definidos em [Akdeniz et al. 2014] para transmissões em $28 \mathrm{GHz}$. A largura de banda é de $1 \mathrm{GHz}$, a potência de transmissão é de $30 \mathrm{dBm}$, o limiar de SNR para a detecção do PSS é de $-5 \mathrm{dBm}$, o limiar de SNR para a recepção na fase de dados é de $20 \mathrm{~dB}$. Além disso, considera-se que o intervalo entre a transmissão das sondas PSS $\left(t_{p e r}\right)$ para o teste de cada combinação de feixe é de $1 \mathrm{~ms}$, conforme considerado por [Barati et al. 2016].

Para os algoritmos que utilizam geolocalização, considerou-se um erro de geolocalização dado por uma distribuição normal com média $\mu$ e variância $\sigma$, conforme a análise estatística de dados experimentais apresentados em [Vaitl et al. 2010]. Foram es- 


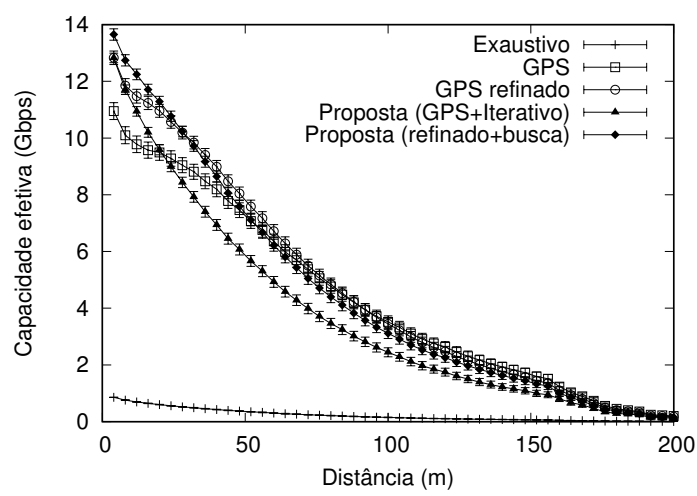

(a) Erro baixo de geolocalização.

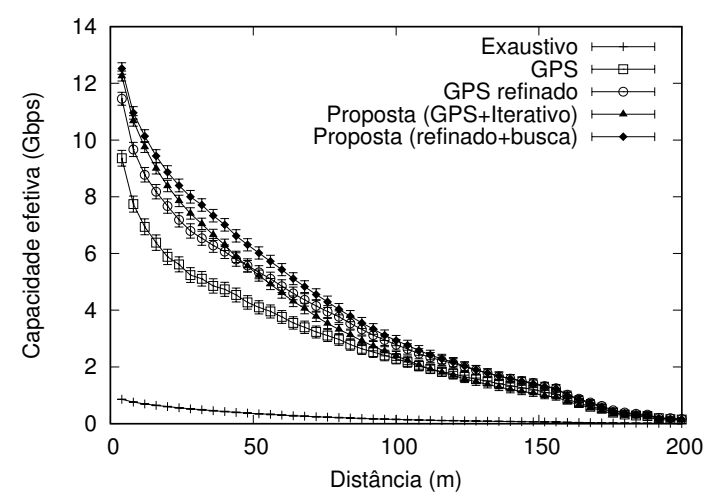

(b) Erro alto de geolocalização.

Figura 9. Capacidade efetiva em função da distância com $t_{\text {frame }}=200 \mathrm{~ms}$.

colhidos dois casos extremos de parametrização para o erro de acordo com os resultados experimentais em [Vaitl et al. 2010]: erro baixo, onde $\mu=5 \mathrm{~m}$ e $\sigma=15 \mathrm{~m}$, e erro alto, onde $\mu=10 \mathrm{~m}$ e $\sigma=60 \mathrm{~m}$.

Nos gráficos apresentados a seguir serão mostradas três métricas de desempenho. A primeira é a capacidade efetiva, conforme explicado na Seção 4. A segunda é a duração do acesso inicial, que é dado pelo tempo médio gasto com o procedimento de acesso inicial por cada algoritmo. E por fim, temos a capacidade nominal que é o valor absoluto de taxa de transmissão que cada algoritmo obtém.

\subsection{Resultados}

No primeiro conjunto de gráficos das Figuras 9, 10 e 11, têm-se os resultados para a métrica capacidade efetiva dos diferentes algoritmos em função da distância entre USR e EBmm em diferentes configurações de $t_{\text {Frame }}$ e erro de geolocalização. Inicialmente, podemos observar o impacto causado pelo erro de geolocalização no desempenho dos algoritmos. A menos da busca exaustiva, que não utiliza informação de geolocalização, pode-se perceber que todos os demais algoritmos têm o desempenho reduzido com o aumento do erro. Isso se justifica pois com estes algoritmos a EBmm tenta encontrar a melhor combinação de feixes utilizando apenas a geolocalização com erro informada ou fazendo algum tipo de varredura limitada à direção indicada pela geolocalização. Quando o erro é alto, esses algoritmos têm a tendência a tomar decisões menos eficientes.

Ainda a respeito do impacto do erro de geolocalização, podemos ver que os dois algoritmos propostos neste trabalho têm o desempenho menos prejudicado pelo aumento do erro do que as propostas já existentes na literatura. Isso ocorre porque as propostas são capazes de fazer uma varredura num espaço angular maior do que o GP S e o GPS refinado. Logo, mesmo que uma localização errada do USR seja repassada para a EBmm, aumenta-se a probabilidade de que um feixe na direção da localização correta seja testado. Isso é uma característica importante dos mecanismos de acesso inicial propostos, que mostra que as propostas têm desempenho mais robusto mesmo em cenários desafiadores com erro de geolocalização elevados.

Outra observação interessante sobre este conjunto de resultados é a respeito do impacto do $t_{\text {Frame }}$. Pode-se perceber que o algoritmo mais influenciado pelo $t_{\text {Frame }}$ é 


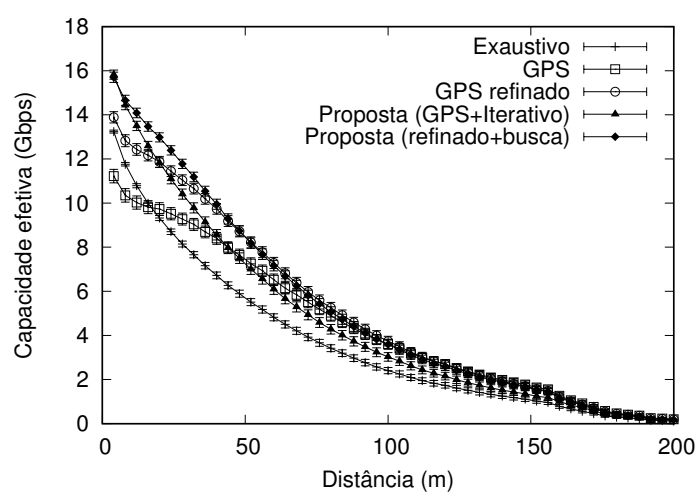

(a) Erro baixo de geolocalização.

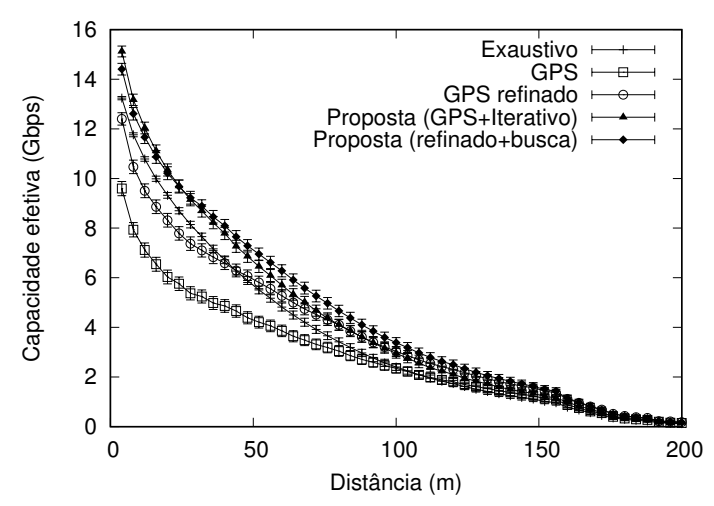

(b) Erro alto de geolocalização.

Figura 10. Capacidade efetiva em função da distância com $t_{\text {frame }}=500 \mathrm{~ms}$.

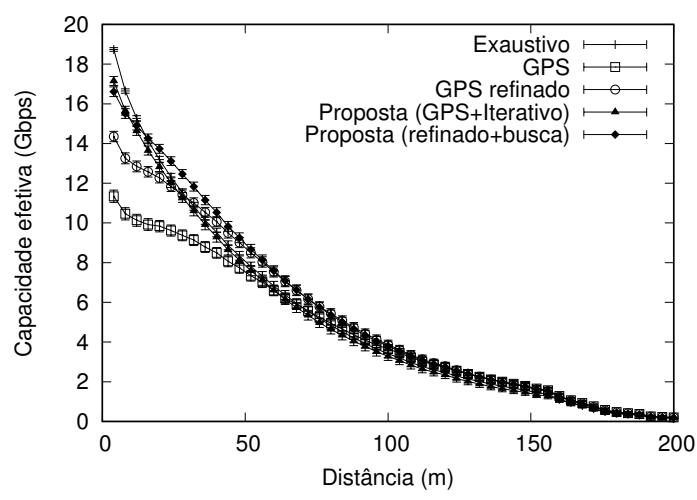

(a) Erro baixo de geolocalização.

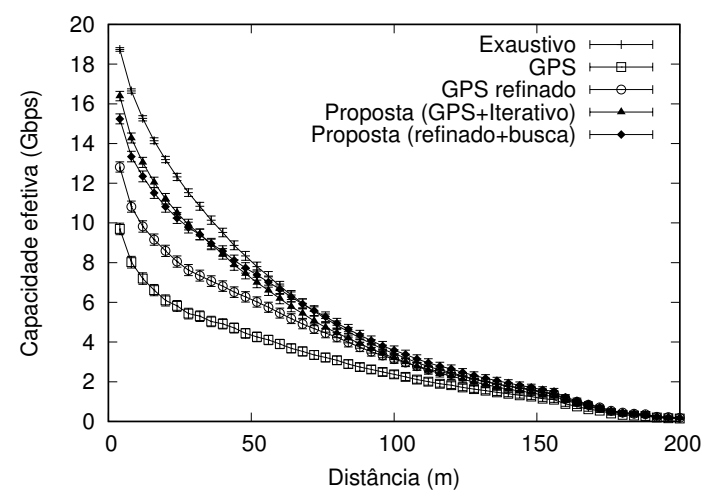

(b) Erro alto de geolocalização.

Figura 11. Capacidade efetiva em função da distância com $t_{\text {frame }}=1500 \mathrm{~ms}$.

o Exaustivo. Isso ocorre porque, apesar de obter a melhor capacidade nominal (Figura 12b), este algoritmo possui um atraso elevado, causado pela necessidade de testar todas as combinações de feixe. A Figura 12a, que apresenta o tempo de acesso inicial em função da distância no cenário com $t_{\text {Frame }}=500 \mathrm{~ms}$ e erro de GPS alto, mostra que o Exaustivo apresenta os piores resultados para o atraso. Apesar disso, quando consideramos a métrica capacidade efetiva, em cenários onde o $t_{\text {Frame }}$ é grande, o Exaust i vo apresenta bons resultados, pois a frequência com que se realiza o acesso inicial é baixa.

Por fim, comparando o desempenho das duas propostas pode-se perceber que, em cenários de erro de GPS alto, o GPS+Iterativo tem o desempenho prejudicado para distâncias grandes. Como este algoritmo utiliza um feixe mais aberto em sua primeira fase, o que reduz o ganho da antena, com o aumento da distância, aumenta também a probabilidade das sondas PSS não serem detectadas pelo USR. Isso fica comprovado pela elevação do tempo de acesso inicial em função da distância na Figura 12a.

\section{Conclusão}

O acesso inicial em ROmm é uma tarefa importante e que pode influenciar no desempenho da rede. Alguns trabalhos que estudam o problema consideram o uso da geolocalização do USR para acelerar esta tarefa, mas desconsideram possíveis impre- 


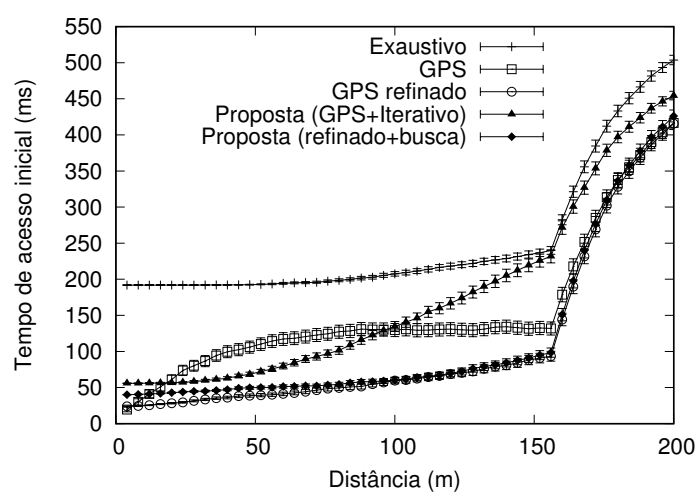

(a) Tempo de acesso inicial

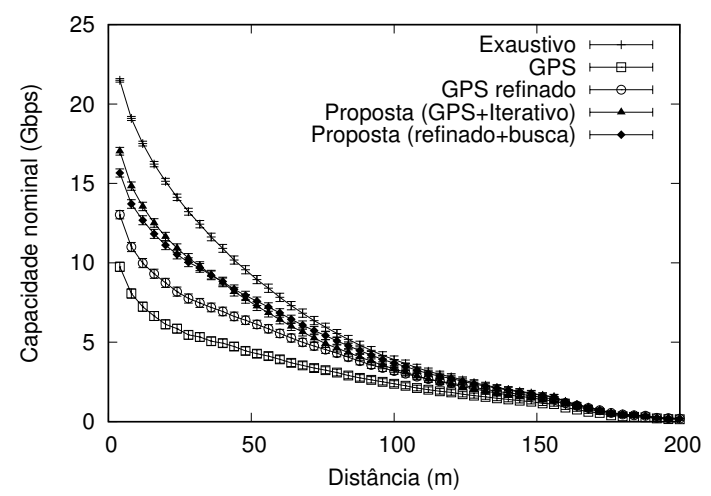

(b) Capacidade nominal.

Figura 12. Tempo de acesso inicial e capacidade nominal para $t_{\text {Frame }}=500 \mathrm{~ms} \mathbf{e}$ erro alto de geolocalização.

cisões nesta informação. Neste trabalho foi proposto um modelo para a análise do impacto do acesso inicial no desempenho da ROmm e dois novos algoritmos de BF que visam lidar com os erros de geolocalização. Os resultados obtidos com a avaliação de desempenho do algoritmo proposto mostram que ele possui maior imunidade a erros de geolocalização que o estado da arte na maioria dos cenários avaliados. Este resultado é causado principalmente pelo fato das propostas cobrirem uma maior área angular no entorno da posição indicada pela geolocalização.

Em trabalhos futuros pretendemos estender esta análise de desempenho para cenários não considerados neste trabalho e propor novos algoritmos que sejam adaptativos ao erro estimado de geolocalização, à frequência com que o acesso inicial é realizado e também à distância estimada entre EBmm e USR.

\section{Referências}

Akdeniz, M. R., Liu, Y., Samimi, M. K., Sun, S., Rangan, S., Rappaport, T. S., and Erkip, E. (2014). Millimeter wave channel modeling and cellular capacity evaluation. IEEE Journal on Selected Areas in Communications, 32:1164-1179.

Bai, T. and Heath, R. W. (2014). Analysis of self-body blocking effects in millimeter wave cellular networks. In 48th Asilomar Conference on Signals, Systems and Computers, pages 1921-1925.

Balanis, C. A. (2016). The Antenna Theory Analysis and Design Book. John Wiley \& Sons, Inc., 4th edition.

Barati, C. N., Hosseini, S. A., Mezzavilla, M., Amiri-Eliasi, P., Rangan, S., Korakis, T., Panwar, S. S., and Zorzi, M. (2015a). Directional initial access for millimeter wave cellular systems. In 49th Asilomar Conference on Signals, Systems and Computers, pages 307-311.

Barati, C. N., Hosseini, S. A., Mezzavilla, M., Korakis, T., Panwar, S. S., Rangan, S., and Zorzi, M. (2016). Initial access in millimeter wave cellular systems. IEEE Transactions on Wireless Communications, 15(12):7926-7940. 
Barati, C. N., Hosseini, S. A., Rangan, S., Liu, P., Korakis, T., Panwar, S. S., and Rappaport, T. S. (2015b). Directional cell discovery in millimeter wave cellular networks. IEEE Transactions on Wireless Communications, 14(12):6664-6678.

Capone, A., Filippini, I., Sciancalepore, V., and Tremolada, D. (2015). Obstacle avoidance cell discovery using mm-waves directive antennas in 5G networks. In IEEE 26th Annual International Symposium on Personal, Indoor, and Mobile Radio Communications (PIMRC), pages 2349-2353.

Giordani, M., Mezzavilla, M., Barati, C. N., Rangan, S., and Zorzi, M. (2016a). Comparative analysis of initial access techniques in $5 \mathrm{G}$ mmwave cellular networks. In Annual Conference on Information Science and Systems (CISS), pages 268-273.

Giordani, M., Mezzavilla, M., and Zorzi, M. (2016b). Initial access in 5G mmwave cellular networks. IEEE Communications Magazine, 54(11):40-47.

Menard, T. and Miller, J. (2011). Comparing the GPS capabilities of the iPhone 4 and iPhone 3G for vehicle tracking using FreeSim Mobile. In IEEE Intelligent Vehicles Symposium (IV), pages 278-283.

Menard, T., Miller, J., Nowak, M., and Norris, D. (2011). Comparing the GPS capabilities of the Samsung Galaxy S, Motorola Droid X, and the Apple iPhone for vehicle tracking using FreeSim Mobile. In 14th International IEEE Conference on Intelligent Transportation Systems (ITSC), pages 985-990.

Peng, H., Moriwaki, K., and Suegara, Y. (2016). Macro-controlled beam database-based beamforming protocol for LTE-WiGig aggregation in millimeter-wave heterogeneous networks. In IEEE 83rd Vehicular Technology Conference (VTC Spring), pages 1-6.

Rangan, S., Rappaport, T. S., and Erkip, E. (2014). Millimeter-wave cellular wireless networks: Potentials and challenges. Proceedings of the IEEE, 102(3):366-385.

Rappaport, T. S. (1996). Wireless communications: Principles and practice. Prentice Hall.

Rappaport, T. S., Sun, S., Mayzus, R., Zhao, H., Azar, Y., Wang, K., Wong, G. N., Schulz, J. K., Samimi, M., and Gutierrez, F. (2013). Millimeter wave mobile communications for 5G cellular: It will work! IEEE Access, 1:335-349.

Renfro, B. A., Stein, M., Boeker, N., and Terry, A. (2018). An analysis of global positioning system (GPS) standard positioning service (SPS) performance for 2017. https://www.gps.gov/systems/gps/performance/ 2017-GPS-SPS-performance-analysis .pdf. Último acesso: 22/12/2018.

Shokri-Ghadikolaei, H., Fischione, C., Fodor, G., Popovski, P., and Zorzi, M. (2015). Millimeter wave cellular networks: A mac layer perspective. IEEE Transactions on Communications, 63(10):3437-3458.

Vaitl, C., Kunze, K., and Lukowicz, P. (2010). Does on-body location of a GPS receiver matter? In International Conference on Body Sensor Networks, pages 219-221.

Wei, L., Li, Q., and Wu, G. (2017). Exhaustive, iterative and hybrid initial access techniques in mmwave communications. In IEEE Wireless Communications and Networking Conference (WCNC), pages 1-6. 